\title{
Primordial Uteroplacental Circulation
}

National Cancer Institute

\section{Source}

National Cancer Institute. Primordial Uteroplacental Circulation. NCI Thesaurus. Code C34258.

Blood flow from lacunar networks that establishes the maternal-placental circulation approximately at the end of the third week of gestation. 